\title{
The Formalization of Decision-Free Petri Net
}

\author{
Pratima K. Shah \\ Shinshu University \\ Nagano, Japan
}

\author{
Pauline N. Kawamoto ${ }^{1}$ \\ Shinshu University \\ Nagano, Japan
}

\author{
Mariusz Gierd ${ }^{2}$ \\ University of Białystok \\ Poland
}

\begin{abstract}
Summary. In this article we formalize the definition of Decision-Free Petri Net (DFPN) presented in [19]. Then we formalize the concept of directed path and directed circuit nets in Petri nets to prove properties of DFPN. We also present the definition of firing transitions and transition sequences with natural numbers marking that always check whether transition is enabled or not and after firing it only removes the available tokens (i.e., it does not remove from zero number of tokens). At the end of this article, we show that the total number of tokens in a circuit of decision-free Petri net always remains the same after firing any sequences of the transition.
\end{abstract}

MSC: 68Q60 68Q85 03B35

Keywords: specification and verification of discrete systems; Petri net;

MML identifier: PETRI_DF, version: 8.1.02 5.22.1199

The notation and terminology used in this paper have been introduced in the following articles: [1], 44, [17, [14], 8], [5], 6], [15], [12, [3], 9], [10], 20], [11, [13], [18, and [7].

\section{Preliminaries}

From now on $N$ denotes a place/transition net structure, $P$ denotes a Petri net, and $i$ denotes a natural number.

\footnotetext{
${ }^{1}$ This work was supported in part by JSPS Kakenhi grant number 2230028502.

${ }^{2}$ This work was supported in part by the University of Bialystok grant BST225 Database of mathematical texts checked by computer.

(C) 2014 University of Białystok
CC-BY-SA License ver. 3.0 or later ISSN 1426-2630(Print), 1898-9934(Online)
} 
Now we state the propositions:

(1) Let us consider natural numbers $x, y$ and a finite sequence $f$. Suppose

(i) $f_{\lfloor 1}$ is one-to-one, and

(ii) $1<x \leqslant \operatorname{len} f$, and

(iii) $1<y \leqslant \operatorname{len} f$, and

(iv) $f(x)=f(y)$.

Then $x=y$.

(2) Let us consider a non empty set $D$ and a non empty finite sequence $f$ of elements of $D$. If $f$ is circular, then $f(1)=f(\operatorname{len} f)$.

Let $D$ be a non empty set and $a, b$ be elements of $D$. Let us observe that $\langle a, b, a\rangle$ is circular as a finite sequence of elements of $D$.

Now we state the proposition:

(3) Let us consider objects $a, b$. If $a \neq b$, then $\langle a, b, a\rangle$ is almost one-to-one.

Let $X$ be a set, $Y$ be a non empty set, $P_{1}$ be a finite subset of $X$, and $M_{1}$ be a function from $X$ into $Y$.

An enumeration of $M_{1}$ and $P_{1}$ is a finite sequence of elements of $Y$ and is defined by

(Def. 1) (i) len $i t=$ len the enumeration of $P_{1}$ and for every $i$ such that $i \in$ dom it holds it $(i)=M_{1}$ (the enumeration of $\left.P_{1}(i)\right)$, if $P_{1}$ is not empty,

(ii) it $=\varepsilon_{Y}$, otherwise.

The functor $\mathrm{PN}_{0}$ yielding a Petri net is defined by the term

(Def. 2) $\left\langle\{0\},\{1\}, \Omega_{\{1\}}(\{0\}), \Omega_{\{0\}}(\{1\})\right\rangle$.

Let us consider $N$. We introduce the places and transitions of $N$ as a synonym of Elements $(N)$.

Let us consider $P$. Let us note that the places and transitions of $P$ is non empty.

In the sequel $f_{1}$ denotes a finite sequence of elements of the places and transitions of $P$.

Let us consider $P$ and $f_{1}$. The functors: the places of $f_{1}$ and the transitions of $f_{1}$ yielding finite subsets of $P$ are defined by terms,

(Def. 3) $\left\{p\right.$, where $p$ is a place of $\left.P: p \in \operatorname{rng} f_{1}\right\}$,

(Def. 4) $\left\{t\right.$, where $t$ is a transition of $\left.P: t \in \operatorname{rng} f_{1}\right\}$, respectively. 


\section{The Number of Tokens in a Circuit}

Let us consider $N$. The markings of $N$ yielding a non empty set of functions from the carrier of $N$ to $\mathbb{N}$ is defined by the term

(Def. 5) $\mathbb{N}^{\alpha}$, where $\alpha$ is the carrier of $N$.

A marking of $N$ is an element of the markings of $N$. Let $P_{1}$ be a finite subset of $N$ and $M_{1}$ be a marking of $N$. The number of tokens of $P_{1}$ and $M_{1}$ yielding an element of $\mathbb{N}$ is defined by the term

(Def. 6) $\quad \sum$ the enumeration of $M_{1}$ and $P_{1}$.

\section{Decision-Free Petri Net Concept and Properties of Circuits in PETRI Nets}

Let $I$ be a Petri net. We say that $I$ is decision-free-like if and only if

(Def. 7) Let us consider a place $s$ of $I$. Then

(i) there exists a transition $t$ of $I$ such that $\langle t, s\rangle \in$ the T-S arcs of $I$, and

(ii) for every transitions $t_{1}, t_{2}$ of $I$ such that $\left\langle t_{1}, s\right\rangle,\left\langle t_{2}, s\right\rangle \in$ the T-S arcs of $I$ holds $t_{1}=t_{2}$, and

(iii) there exists a transition $t$ of $I$ such that $\langle s, t\rangle \in$ the S-T arcs of $I$, and

(iv) for every transitions $t_{1}, t_{2}$ of $I$ such that $\left\langle s, t_{1}\right\rangle,\left\langle s, t_{2}\right\rangle \in$ the S-T arcs of $I$ holds $t_{1}=t_{2}$.

Let us consider $P$. Let $I$ be a finite sequence of elements of the places and transitions of $P$. We say that $I$ is directed path if and only if

(Def. 8) (i) len $I \geqslant 3$, and

(ii) len $I \bmod 2=1$, and

(iii) for every $i$ such that $i \bmod 2=1$ and $i+1<\operatorname{len} I$ holds $\langle I(i)$, $I(i+1)\rangle \in$ the S-T arcs of $P$ and $\langle I(i+1), I(i+2)\rangle \in$ the T-S arcs of $P$, and

(iv) $I($ len $I) \in$ the carrier of $P$.

Now we state the proposition:

(4) Let us consider a finite sequence $f_{1}$ of elements of the places and transitions of $\mathrm{PN}_{0}$. Suppose $f_{1}=\langle 0,1,0\rangle$. Then $f_{1}$ is directed path. Proof: $f_{1}$ is directed path by [2, (13)], [4, (45)].

Let us consider $P$. Observe that every finite sequence of elements of the places and transitions of $P$ which is directed path is also non empty.

Let $I$ be a Petri net. We say that $I$ has directed path if and only if 
(Def. 9) There exists a finite sequence $f_{1}$ of elements of the places and transitions of $I$ such that $f_{1}$ is directed path.

Let us consider $P$. We say that $P$ has directed circuit if and only if

(Def. 10) There exists $f_{1}$ such that $f_{1}$ is directed path, circular, and almost oneto-one.

One can verify that $\mathrm{PN}_{0}$ is decision-free-like and Petri-like and has directed circuit and there exists a Petri net which is Petri-like and decision-free-like and has directed circuit and every Petri net which has directed circuit has also directed path and there exists a Petri net which has directed path.

Let $D_{1}$ be a Petri net with directed path. Let us note that there exists a finite sequence of elements of the places and transitions of $D_{1}$ which is directed path.

From now on $D_{1}$ denotes a Petri net with directed path and $d$ denotes a directed path finite sequence of elements of the places and transitions of $D_{1}$.

Now we state the propositions:

(5) $\langle d(1), d(2)\rangle \in$ the S-T $\operatorname{arcs}$ of $D_{1}$.

(6) $\langle d($ len $d-1), d($ len $d)\rangle \in$ the T-S $\operatorname{arcs}$ of $D_{1}$.

From now on $D_{1}$ denotes a Petri-like Petri net with directed path and $d$ denotes a directed path finite sequence of elements of the places and transitions of $D_{1}$.

Now we state the proposition:

(7) If $d(i) \in$ the places of $d$ and $i \in \operatorname{dom} d$, then $i \bmod 2=1$. Proof: Consider $p$ being a place of $D_{1}$ such that $p=d(i)$ and $p \in \operatorname{rng} d . i \bmod 2=$ 1 by [2, (21)], [16, (25)], [7, (87)].

Let us assume that $d(i) \in$ the transitions of $d$ and $i \in \operatorname{dom} d$. Now we state the propositions:

(8) $i \bmod 2=0$. Proof: $\langle d(\operatorname{len} d-1), d(\operatorname{len} d)\rangle \in$ the T-S arcs of $D_{1}$. Consider $t$ being a transition of $D_{1}$ such that $t=d(i)$ and $t \in \operatorname{rng} d$. $i \neq$ len $d$ by [7, (87)]. $i+1 \neq$ len $d$ by [7, (87)], [2, (11)], [16, (25)], [5, (3)].

(9) (i) $\langle d(i-1), d(i)\rangle \in$ the S-T arcs of $D_{1}$, and

(ii) $\langle d(i), d(i+1)\rangle \in$ the T-S $\operatorname{arcs}$ of $D_{1}$.

Proof: $\langle d($ len $d-1), d($ len $d)\rangle \in$ the T-S arcs of $D_{1}$. Consider $t$ being a transition of $D_{1}$ such that $t=d(i)$ and $t \in \operatorname{rng} d . i \neq$ len $d$ by [7, (87)].

Now we state the proposition:

(10) Suppose $d(i) \in$ the places of $d$ and $1<i<$ len $d$. Then

(i) $\langle d(i-2), d(i-1)\rangle \in$ the S-T arcs of $D_{1}$, and

(ii) $\langle d(i-1), d(i)\rangle \in$ the T-S arcs of $D_{1}$, and

(iii) $\langle d(i), d(i+1)\rangle \in$ the S-T arcs of $D_{1}$, and 
(iv) $\langle d(i+1), d(i+2)\rangle \in$ the T-S arcs of $D_{1}$, and

(v) $3 \leqslant i$.

Proof: $i \bmod 2=1$. $\langle d(\operatorname{len} d-1), d(\operatorname{len} d)\rangle \in$ the T-S arcs of $D_{1} \cdot\langle d(1)$, $d(2)\rangle \in$ the S-T arcs of $D_{1}$. Consider $p$ being a place of $D_{1}$ such that $p=d(i)$ and $p \in \operatorname{rng} d . i+1 \neq \operatorname{len} d$ by [7, (87)]. $2 \neq i$ by [7, (87)].

\section{Firable and Firing Conditions for Transitions and Transition Sequences with Natural Marking}

From now on $M_{1}$ denotes a marking of $P, t$ denotes a transition of $P$, and $Q, Q_{1}$ denote finite sequences of elements of the carrier' of $P$.

Let us consider $P, M_{1}$, and $t$. We say that $t$ is firable at $M_{1}$ if and only if

(Def. 11) Let us consider a natural number $m$. If $\left.m \in M_{1}{ }^{\circ}{ }^{*}\{t\}\right)$, then $m>0$.

The functor Firing $\left(t, M_{1}\right)$ yielding a marking of $P$ is defined by

(Def. 12) (i) for every place $s$ of $P$, if $s \in{ }^{*}\{t\}$ and $s \notin \overline{\{t\}}$, then it $(s)=M_{1}(s)-1$ and if $s \in \overline{\{t\}}$ and $s \notin *\{t\}$, then it $(s)=M_{1}(s)+1$ and if $s \in{ }^{*}\{t\}$ and $s \in \overline{\{t\}}$ or $s \notin^{*}\{t\}$ and $s \notin \overline{\{t\}}$, then it $(s)=M_{1}(s)$, if $t$ is firable at $M_{1}$,

(ii) it $=M_{1}$, otherwise.

Let us consider $Q$. We say that $Q$ is firable at $M_{1}$ if and only if

(Def. 13) (i) $Q=\emptyset$, or

(ii) there exists a finite sequence $M$ of elements of the markings of $P$ such that len $Q=\operatorname{len} M$ and $Q_{1}$ is firable at $M_{1}$ and $M_{1}=\operatorname{Firing}\left(Q_{1}, M_{1}\right)$ and for every $i$ such that $i<\operatorname{len} Q$ and $i>0$ holds $Q_{i+1}$ is firable at $M_{i}$ and $M_{i+1}=\operatorname{Firing}\left(Q_{i+1}, M_{i}\right)$.

The functor Firing $\left(Q, M_{1}\right)$ yielding a marking of $P$ is defined by

(Def. 14) (i) it $=M_{1}$, if $Q=\emptyset$,

(ii) there exists a finite sequence $M$ of elements of the markings of $P$ such that len $Q=$ len $M$ and $i t=M_{\text {len } M}$ and $M_{1}=\operatorname{Firing}\left(Q_{1}, M_{1}\right)$ and for every $i$ such that $i<\operatorname{len} Q$ and $i>0$ holds $M_{i+1}=\operatorname{Firing}\left(Q_{i+1}, M_{i}\right)$, otherwise.

Now we state the propositions:

(11) Firing $\left(t, M_{1}\right)=$ Firing $\left(\langle t\rangle, M_{1}\right)$.

(12) $t$ is firable at $M_{1}$ if and only if $\langle t\rangle$ is firable at $M_{1}$.

(13) $\operatorname{Firing}\left(Q^{\frown} Q_{1}, M_{1}\right)=\operatorname{Firing}\left(Q_{1}, \operatorname{Firing}\left(Q, M_{1}\right)\right)$.

(14) If $Q^{\frown} Q_{1}$ is firable at $M_{1}$, then $Q_{1}$ is firable at $\operatorname{Fining}\left(Q, M_{1}\right)$ and $Q$ is firable at $M_{1}$. 


\section{The Theorem Stating that the Number of Tokens in a Circuit Remains the Same After any Firing Sequences}

Now we state the proposition:

(15) Let us consider a Petri-like decision-free-like Petri net $D_{1}$ with directed path, a directed path finite sequence $d$ of elements of the places and transitions of $D_{1}$, and a transition $t$ of $D_{1}$. Suppose

(i) $d$ is circular, and

(ii) there exists a place $p_{1}$ of $D_{1}$ such that $p_{1} \in$ the places of $d$ and $\left\langle p_{1}\right.$, $t\rangle \in$ the S-T arcs of $D_{1}$ or $\left\langle t, p_{1}\right\rangle \in$ the T-S arcs of $D_{1}$.

Then $t \in$ the transitions of $d$. The theorem is a consequence of (7), (5), (6), and (2).

A decision-free Petri net is a Petri-like decision-free-like Petri net with directed circuit. Let $D_{1}$ be a Petri net with directed circuit. Observe that there exists a finite sequence of elements of the places and transitions of $D_{1}$ which is directed path, circular, and almost one-to-one.

A circuit of places and transitions of $D_{1}$ is a directed path circular almost one-to-one finite sequence of elements of the places and transitions of $D_{1}$. Now we state the propositions:

(16) Let us consider a decision-free Petri net $D_{1}$, a circuit $d$ of places and transitions of $D_{1}$, a marking $M_{1}$ of $D_{1}$, and a transition $t$ of $D_{1}$. Then the number of tokens of the places of $d$ and $M_{1}=$ the number of tokens of the places of $d$ and Firing $\left(t, M_{1}\right)$. The theorem is a consequence of (6), (5), (8), (2), (9), (1), (10), and (15).

(17) Let us consider a decision-free Petri net $D_{1}$, a circuit $d$ of places and transitions of $D_{1}$, a marking $M_{1}$ of $D_{1}$, and a finite sequence $Q$ of elements of the carrier' of $D_{1}$. Then the number of tokens of the places of $d$ and $M_{1}=$ the number of tokens of the places of $d$ and $\operatorname{Firing}\left(Q, M_{1}\right)$. The theorem is a consequence of (16).

\section{REFERENCES}

[1] Grzegorz Bancerek. Cardinal numbers Formalized Mathematics, 1(2):377-382, 1990.

[2] Grzegorz Bancerek. The fundamental properties of natural numbers Formalized Mathematics, 1(1):41-46, 1990.

[3] Grzegorz Bancerek. The ordinal numbers. Formalized Mathematics, 1(1):91-96, 1990.

[4] Grzegorz Bancerek and Krzysztof Hryniewiecki. Segments of natural numbers and finite sequences. Formalized Mathematics, 1(1):107-114, 1990.

[5] Czesław Byliński. Functions and their basic properties Formalized Mathematics, 1(1): 55-65, 1990.

[6] Czesław Byliński. Functions from a set to a set. Formalized Mathematics, 1(1):153-164, 1990.

[7] Czesław Byliński. Some basic properties of sets Formalized Mathematics, 1(1):47-53, 1990. 
[8] Agata Darmochwał. Finite sets Formalized Mathematics, 1(1):165-167, 1990.

[9] Pauline N. Kawamoto, Yasushi Fuwa, and Yatsuka Nakamura. Basic Petri net concepts Formalized Mathematics, 3(2):183-187, 1992.

[10] Andrzej Kondracki. Basic properties of rational numbers Formalized Mathematics, 1(5): 841-845, 1990.

[11] Jarosław Kotowicz. Functions and finite sequences of real numbers Formalized Mathematics, 3(2):275-278, 1992.

[12] Robert Milewski. Subsequences of almost, weakly and poorly one-to-one finite sequences Formalized Mathematıcs, 13(2):227-233, 2005.

[13] Karol Pąk. Continuity of barycentric coordinates in Euclidean topological spaces. For malized Mathematics, 19(3):139-144, 2011. doi 10.2478/v10037-011-0022-5.

[14] Andrzej Trybulec. On the decomposition of finite sequences Formalized Mathematics, 5 (3):317-322, 1996.

[15] Michał J. Trybulec. Integers. Formalized Mathematics, 1(3):501-505, 1990.

[16] Wojciech A. Trybulec. Non-contiguous substrings and one-to-one finite sequences Formalized Mathematics, 1(3):569-573, 1990.

[17] Wojciech A. Trybulec. Pigeon hole principle Formalized Mathematics, 1(3):575-579, 1990.

[18] Zinaida Trybulec. Properties of subsets. Formalized Mathematics, 1(1):67-71, 1990.

[19] Jiacun Wang. Timed Petri Nets, Theory and Application. Kluwer Academic Publishers, 1998.

[20] Edmund Woronowicz. Relations and their basic properties Formalized Mathematics, 1 (1):73-83, 1990.

Received March 31, 2014 